\title{
Does conventional freezing affect sperm DNA fragmentation?
}

\author{
Minh Tam Le ${ }^{1,2}$, Thai Thanh Thi Nguyen², Tung Thanh Nguyen ${ }^{3}$, Trung Van Nguyen², Tam An Thi Nguyen², Quoc Huy Vu Nguyen', \\ Thanh Ngoc Cao ${ }^{1,2}$
}

${ }^{1}$ Department of Obstetrics and Gynecology, ${ }^{2}$ Center for Reproductive Endocrinology and Infertility, and ${ }^{3}$ Department of Histology and Embryology, Hue University of Medicine and Pharmacy, Hue University, Hue, Vietnam

\begin{abstract}
Objective: Sperm cryopreservation has been widely used in assisted reproductive technology, as it offers great potential for the treatment of some types of male infertility. However, cryopreservation may result in changes in membrane lipid composition and acrosome status, as well as reductions in sperm motility and viability. This study aimed to evaluate sperm DNA fragmentation damage caused by conventional freezing using the sperm chromatin dispersion test.

Methods: In total, 120 fresh human semen samples were frozen by conventional methods, using SpermFreeze Solution as a cryoprotectant. Routine semen analysis and a Halosperm test (using the Halosperm kit) were performed on each sample before freezing and after thawing. Semen parameters and sperm DNA fragmentation were compared between these groups.

Results: There was a significant decrease in sperm progressive motility, viability, and normal morphology after conventional freezing $(32.78 \%$, $79.58 \%$, and $3.87 \%$ vs. $16 \%, 55.99 \%$, and $2.55 \%$, respectively). The sperm head, midpiece, and tail defect rate increased slightly after freezing. Furthermore, the DNA fragmentation index (DFI) was significantly higher after thawing than before freezing (19.21\% prior to freezing vs. $22.23 \%$ after thawing). Significant increases in the DFI after cryopreservation were observed in samples with both normal and abnormal motility and morphology, as well as in those with normal viability.

Conclusion: Conventional freezing seems to damage some sperm parameters, in particular causing a reduction in sperm DNA integrity.
\end{abstract}

Keywords: Cryopreservation; DNA fragmentation; Freezing; Halosperm test; Spermatozoa

\section{Introduction}

Infertility is a major problem in modern life. Male infertility accounts for about $50 \%$ of cases of infertility experienced by couples, and $90 \%$ of male infertility is due to sperm abnormalities [1]. Fortunately, sperm cryopreservation has been widely used in assisted reproductive technology (ART), with great potential for infertility treatment. Numerous cryopreservation methods exist, but all involve cooling,

Received: Jan 14, 2019· Revised: Apr 15, 2019· Accepted: Apr 16, 2019

Corresponding author: Minh Tam Le

Department of Obstetrics and Gynecology, Hue University of Medicine and Pharmacy, Hue University, 06 Ngo Quyen St., Hue City, Vietnam

Tel:+84-989228779 Fax:+84-2343822873 E-mail: leminhtam@huemed-univ.edu.vn

This is an Open Access article distributed under the terms of the Creative Commons Attribution Non-Commercial License (http://creativecommons.org/licenses/by-nc/4.0/) which permits unrestricted non-commercial use, distribution, and reproduction in any medium, provided the original work is properly cited. freezing, and thawing. Although the reduction of sperm motility, morphology, and viability after thawing has been studied widely [2], controversy remains regarding how sperm DNA integrity is affected by various cryopreservation methods. Many studies have shown that these processes may damage sperm function [3]. For example, Hammadeh et al. [4] found that cryopreservation resulted in changes in membrane lipid composition and acrosome status, reductions in sperm motility and viability, and sperm DNA fragmentation [5]. However, other studies have found that cryopreservation did not damage sperm DNA [6]. Thus, the question of whether cryopreservation affects or causes sperm DNA fragmentation remains unresolved.

Conventional semen analysis by itself does not adequately address this question because sperm parameters such as $\mathrm{pH}$, volume, concentration, motility, survival rate, and morphology in this routine analysis may not reflect molecular factors. Recently, extensive re- 
search has investigated the subject of human sperm DNA integrity, as well as the mechanisms of sperm DNA damage and its effects on ART outcomes. Several tests are available to evaluate sperm DNA integrity based on measurements of abnormalities in sperm chromatin structure assay (SCSA) or through direct assessments of DNA strand breaks, including the sperm chromatin structure assay, acridine orange test, toluidine blue test, aniline blue test, terminal deoxynucleotidyl transferase dUTP nick end labeling (TUNEL), the in situ nick translation assay, the Comet assay, sperm chromatin dispersion (SCD), or sperm DNA compaction (the CMA3 assay) $[7,8]$. The Halosperm test is an SCD test based on induced condensation, which is directly linked with sperm DNA fragmentation [9]. This test is now performed conveniently using the patented Halosperm kit (Halotech DNA, Madrid, Spain); its application is easy and cost-effective in laboratories in developing countries such as Vietnam.

Controversy exists about both how much harm cryopreservation may inflict on sperm DNA integrity and whether some cryopreservation methods cause more damage than others. Several effective cryopreservation methods have been used, such as slow programmable freezing, conventional freezing, and vitrification. Conventional freezing is the most popular of these methods in Vietnam because it does not require special equipment, and it is convenient, easy to perform, and inexpensive. In general, the conventional freezing procedure involves first immersing the sample in vapors from liquid nitrogen, and then immersing the sample in the liquid itself, at a temperature of $-196^{\circ} \mathrm{C}[10]$.

In recent years, studies have compared conventional freezing to vitrification, a newer technique. Some studies found no significant differences between conventional freezing and vitrification in posttreatment motility, viability, mitochondrial membrane potential, or the percentage of normal sperm morphology [11,12]. In contrast, other researchers found that vitrification yielded superior results compared to conventional rapid freezing in terms of sperm parameters such as motility, viability, DNA structure, acrosome status, and morphology [6,13].

The literature shows that disagreements continue regarding the damaging effects of conventional freezing on DNA integrity. Therefore, it would be meaningful to identify the impact of conventional freezing on sperm DNA fragmentation using the Halosperm test.

\section{Methods}

\section{Study population}

A total of 120 semen samples were randomly collected from the male partners of infertile couples being treated at the Hue Center for Reproductive Endocrinology and Infertility, Hue University of Medicine and Pharmacy, Vietnam, between October 2017 and August 2018. This study was approved by the Ethics Committee of Hue University of Medicine and Pharmacy, Vietnam.

\section{Semen collection and assessment}

Semen samples from patients were collected by masturbation and ejaculation after abstinence for 2-7 days, with the exclusion of samples with cryptozoospermia, azoospermia, or less than 5 million spermatozoa per milliliter. Samples were analyzed and classified according to the 2010 World Health Organization (WHO) guidelines for examination and processing of human semen [14].

\section{Semen analysis \\ 1) Sperm motility}

Sperm motility was classified as progressive motility or nonprogressive motility through an analysis by manual counting under microscopy using a Primo Star microscope (Zeiss, Jena, Germany). The sperm with progressive motility moved actively, either linearly or in a large circle. The sperm with nonprogressive motility either moved in a small circle or were immotile, despite showing clear flagella movement. Sperm samples in which at least $32 \%$ of sperm were classified as showing progressive motility were considered to have normal motility.

\section{2) Sperm vitality}

The percentage of viable sperm was calculated by using the eosin technique to determine whether sperm were dead or live. Dead sperm were stained or partially stained red due to dye exclusion, while live sperm cells were white. According to the WHO criteria, a sample with normal sperm viability was defined as one in which at least $58 \%$ of sperm were live (WHO, 2010) [14].

\section{3) Sperm morphology}

The morphology of the sperm head (shape and size), acrosome region, sperm neck, mid-piece, tail, and cytoplasmic droplets was determined under microscopy using a Primo Star microscope (Zeiss) at $\times 1,000$ power using the Giemsa stain procedure, following the WHO guideline (fifth edition) [14]. At least 200 spermatozoa were counted to calculate the percentage with normal and abnormal morphology. A sperm sample was considered to be morphologically normal if at least $4 \%$ of the sperm cells showed normal morphology (WHO, 2010) [14].

\section{Halosperm test}

The Halosperm test, which is based on the SCD technique for measuring sperm DNA fragmentation, was performed using the Halosperm kit (Halotech DNA), following the manufacturer's protocol [15]. Briefly, a diluted semen sample was mixed with melted agarose and 


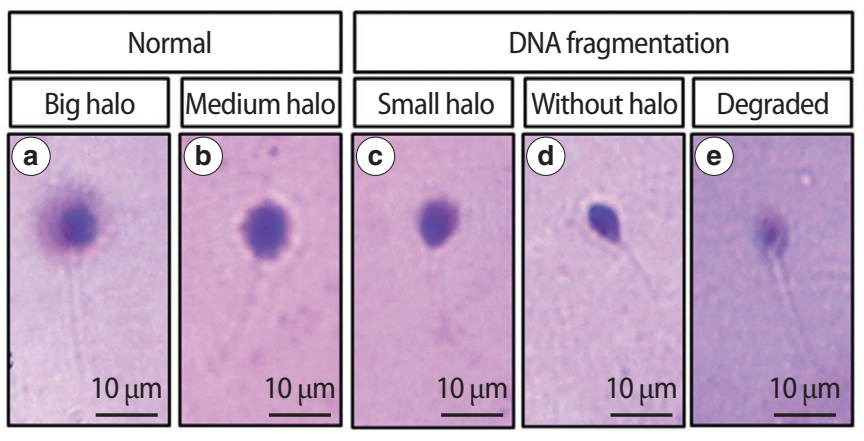

Figure 1. Classification of human sperm DNA fragmentation using Halosperm test. (a) Big/large halo: halo width $\geq$ the diameter of the core, (b) medium halo: small halo $\leq$ medium halo $\leq$ big halo, (c) small halo: halo width $\leq 1 / 3$ of the diameter of the core, (d) without halo: no halo, (e) degraded: no halo and presents a core irregularly or weakly stained.

placed on a slide. After removing the coverslip, the sample was then immersed in $\mathrm{HCl}$ solution for 7 minutes. Before washing with water and ethanol $(70 \%, 90 \%$, and $100 \%)$, the sample was immersed in lysing solution for 25 minutes. The halos of dispersed DNA loops were observed via bright-field microscopy under a $\times 40$ objective using a Primo Star microscope (Zeiss), after staining by the Giemsa procedure. In total, 500 spermatozoa were evaluated in this test. DNA fragmentation was assessed in this manner for both fresh and thawed sperm.

The DNA fragmentation index (DFI), a measure of sperm DNA fragmentation, is a calculation of the percent of sperm affected by DNA damage. It is calculated using the following formula:

$$
\text { DFI }(\%)=\frac{\text { Fragmented }+ \text { degraded }}{\text { Total cells }} \times 100
$$

The index was calculated for the number of sperm observed to exhibit each of the five patterns of DNA loop halos described in Figure 1. Sperm with fragmented DNA were defined as those that failed to produce a halo of dispersed DNA, while sperm with intact DNA manifested such a halo. Spermatozoa were considered to have damaged DNA if the DFI was over 30\% [16]. In this study, we used $30 \%$ as the cutoff value of DFI [15]. The five categories of SCD categories are defined in Figure 1, following previously established criteria [15].

\section{Conventional freezing}

The freezing procedure was modified from the SpermFreeze Solution guideline (REF 10137; Vitrolife, Vastra Frolunda, Sweden). SpermFreeze Solution is a medium consisting of bicarbonate and MOPS buffered with glycerol. Glycerol acts as a permeating cryoprotectant agent (CPA) in the freezing process. This CPA protects the sperm against thermal shock [17].

SpermFreeze Solution was added slowly by drops to the sample in a 1.8 mL Nunc Cryotube vial (catalog No. 375418; Thermo Fisher Scientific, Jiangsu, China) at a ratio of 1:1 and gently mixed after each drop was added. The process took 10 minutes at room temperature. Next, the Cryotube was placed horizontally $3 \mathrm{~cm}$ above the liquid nitrogen surface to minimize the heat difference between the two ends and to increase the surface area in contact with nitrogen vapors. During this process, there is a thermal gradient, and the freezing temperature was estimated to be approximately $-70^{\circ} \mathrm{C},-80^{\circ} \mathrm{C}$, and $-99^{\circ} \mathrm{C}$ [10]. After 15 minutes, the Cryotube was submerged into liquid nitrogen and then stored in a tank at $-196^{\circ} \mathrm{C}$ for at least 1 month before thawing.

\section{Thawing of samples}

Thawing was performed in a manner modified from the description in the SpermFreeze Solution guideline after 1 month's freezing. The Cryotube was removed from the liquid nitrogen and submerged in warm water $\left(37^{\circ} \mathrm{C}\right)$ for 5 minutes. This is an essential step in order to avoid abrupt thermal changes [10]. After using clean paper towels to wipe the Cryotube dry, the post-thaw sperm parameters (motility, vitality, morphology, and DFI) were analyzed.

\section{Statistical analysis}

All analyses were performed using IBM SPSS ver. 20.0 (IBM Corp., Armonk, NY, USA). Data are expressed as mean and standard deviation or as absolute numbers and percentages. The paired-samples $t$ test was used to compare results before freezing and after thawing. The chi-square test was used to compare the ratios of variables. Comparisons between groups were evaluated by the independent-samples $t$-test and analysis of variance (the Tukey test). Although $p<0.05$ was initially considered as the cutoff for statistical significance, we decided to select a cutoff $p$-value of $\leq 0.001$ to ensure results of high significance.

\section{Results}

\section{Characteristics of the study population}

The characteristics of the study population are shown in Table 1. The mean age was $34.48 \pm 5.82$ years, with a range from 24 to 53 years old. Primary infertility was present in $74.2 \%$ of the patients. Their mean duration of infertility was $3.9 \pm 2.3$ years, with a range from 1 to 15 years. $38.3 \%$ of the men were cigarette smokers, and $60.8 \%$ of the men reported regularly drinking alcohol. The mean BMI of these men was $23.4 \pm 2.8 \mathrm{~kg} / \mathrm{m}^{2}$.

\section{Correlations between characteristics of the study population and DFI in fresh semen}

We did not find significant differences in the DFI according to the 
Table 1. Basic characteristics of the study population $(n=120)$

\begin{tabular}{lc}
\hline Characteristics & No. (\%) \\
\hline Age (yr) & \\
$\geq 35$ & $50(41.7)$ \\
$<35$ & $70(58.3)$ \\
Infertility type & \\
Primary & $89(74.2)$ \\
Secondary & $31(25.8)$ \\
Infertility duration (yr) & \\
$>3$ & $60(50)$ \\
$\leq 3$ & $60(50)$ \\
BMl (kg/m²) & \\
$<18.5$ & $5(4.2)$ \\
$18.5-22.9$ & $40(33.3)$ \\
$23-24.9$ & $43(35.8)$ \\
$\geq 25$ & $32(26.7)$ \\
Occupation & \\
Office & $48(40)$ \\
Manual & $32(26.7)$ \\
Others & $40(33.3)$ \\
Smoking & \\
Yes & $46(38.3)$ \\
No & $74(61.7)$ \\
Alcohol consumption & \\
Yes & $73(60.8)$ \\
No & $47(39.2)$ \\
\hline
\end{tabular}

BMI, body mass index. various characteristics of the subject population. When samples were dichotomized into those with intact or non-intact DNA using a DFI cutoff of 30\%, statistical significance was likewise not found according to the various characteristics of the population (Table 2).

\section{Effects of conventional freezing on sperm parameters}

The results of the baseline semen analysis are shown in Table 3. The population in our study had a large range of concentration, progressive motility, and viability. Generally, the fresh semen samples showed no outliers worthy of exclusion. Moreover, the pre-freezing and post-thawing sperm parameter values are displayed in Table 4.

Table 3. Fresh semen analysis $(n=120)$

\begin{tabular}{lcc}
\hline Sperm parameter & Mean \pm SD (range) & $\begin{array}{c}\text { WHO criteria } \\
(2010)\end{array}$ \\
\hline $\mathrm{pH}$ & $7.05 \pm 0.24(6.0-8.0)$ & $\geq 7.2$ \\
Volume $(\mathrm{mL})$ & $1.67 \pm 0.87(0.5-4.0)$ & $\geq 1.5$ \\
Concentration (million/mL) & $33.23 \pm 12.86(5.0-64.0)$ & $\geq 15$ \\
Progressive motility (\%) & $32.78 \pm 12.38(3.0-65.0)$ & $\geq 32$ \\
Viability (\%) & $79.58 \pm 7.3(50.0-94.0)$ & $\geq 58$ \\
Normal morphology (\%) & $3.87 \pm 2.01(1.0-12.0)$ & $\geq 4$ \\
\hline
\end{tabular}

SD, standard deviation; WHO, World Health Organization.

Table 2. Correlations between characteristics of the study population and the DFl in fresh semen $(n=120)$

\begin{tabular}{|c|c|c|c|c|c|}
\hline Characteristics & DFI (\%) & $p$-value & $\mathrm{DFI}>30 \%$ & $\mathrm{DFI} \leq 30 \%$ & $p$-value \\
\hline Age (yr) & & 0.73 & & & 0.51 \\
\hline$\geq 35$ & $19.59 \pm 9.73$ & & $7(14.0)$ & $43(86.0)$ & \\
\hline$<35$ & $18.94 \pm 10.34$ & & $13(18.6)$ & $57(81.4)$ & \\
\hline Infertility type & & 0.52 & & & 0.93 \\
\hline Primary & $19.56 \pm 9.23$ & & $15(16.9)$ & 74 (83.1) & \\
\hline Secondary & $18.21 \pm 12.23$ & & $5(16.1)$ & $26(83.9)$ & \\
\hline Infertility duration (yr) & & 0.69 & & & 0.33 \\
\hline$>3$ & $18.84 \pm 10.19$ & & $8(13.3)$ & $52(86.7)$ & \\
\hline$\leq 3$ & $19.58 \pm 9.99$ & & $12(20.0)$ & $48(80.0)$ & \\
\hline BMI $\left(\mathrm{kg} / \mathrm{m}^{2}\right)$ & & 0.24 & & & 0.56 \\
\hline$<18.5$ & $27.92 \pm 9.15$ & & $2(40.0)$ & $3(60.0)$ & \\
\hline $18.5-22.9$ & $18.16 \pm 9.12$ & & $6(15.0)$ & $34(85.0)$ & \\
\hline $23-24.9$ & $19.17 \pm 10.57$ & & $7(16.3)$ & $36(83.6)$ & \\
\hline$\geq 25$ & $19.21 \pm 10.06$ & & $5(15.6)$ & $27(84.4)$ & \\
\hline Occupation & & 0.59 & & & 0.57 \\
\hline Office & $19.88 \pm 9.58$ & & $8(16.7)$ & $40(83.3)$ & \\
\hline Manual & $19.86 \pm 9.51$ & & $7(21.9)$ & $25(78.1)$ & \\
\hline Others & $17.89 \pm 11.11$ & & $5(12.5)$ & $35(87.5)$ & \\
\hline Geography & & 0.13 & & & 0.57 \\
\hline Urban & $20.79 \pm 10.65$ & & $10(18.9)$ & 43 (81.1) & \\
\hline Rural & $17.96 \pm 9.46$ & & $10(14.9)$ & $57(85.1)$ & \\
\hline Smoking & & 0.54 & & & 0.4 \\
\hline Yes & $18.49 \pm 8.46$ & & $6(13.0)$ & $40(87.0)$ & \\
\hline No & $19.66 \pm 10.96$ & & $14(18.9)$ & $60(81.1)$ & \\
\hline Alcohol consumption & & 0.29 & & & 0.93 \\
\hline Yes & $19.99 \pm 10.55$ & & $12(16.4)$ & $61(83.6)$ & \\
\hline No & $17.99 \pm 9.21$ & & $8(17.0)$ & $39(83.0)$ & \\
\hline
\end{tabular}

Values are presented as mean \pm standard deviation or number (\%). DFI, DNA fragmentation index; BMI, body mass index. 
Table 4. Comparison of sperm parameters prior to freezing and following thawing $(n=120)$

\begin{tabular}{lccc}
\hline Sperm parameter & Before freezing & After thawing & $p$-value \\
\hline Progressive motility (\%) & $32.78 \pm 12.38$ & $16 \pm 9.08$ & $<0.001$ \\
Viability (\%) & $79.58 \pm 7.3$ & $55.99 \pm 8.14$ & $<0.001$ \\
Normal morphology (\%) & $3.87 \pm 2.01$ & $2.55 \pm 1.45$ & $<0.001$ \\
Head defect (\%) & $85.13 \pm 4.54$ & $86.03 \pm 4.66$ & $<0.001$ \\
Midpiece or tail defect (\%) & $64.2 \pm 9.54$ & $65.3 \pm 9.32$ & $<0.001$ \\
DFI (\%) & $19.21 \pm 10.6$ & $22.23 \pm 8.9$ & $<0.001$ \\
\hline
\end{tabular}

Values are presented as mean \pm standard deviation.

DFI, DNA fragmentation index.

Table 5. The effects of conventional freezing on DFI in groups dichotomized by different parameters $(n=120)$

\begin{tabular}{lccc}
\hline Group & \multicolumn{2}{c}{ DFI (\%) } & After thawing \\
\cline { 2 - 3 } & Before freezing & & \\
Motility & & $22.15 \pm 9.56$ & $<.001$ \\
$\quad$ Normal $(\geq 32 \%)$ & $18.38 \pm 9.89$ & $22.31 \pm 8.14$ & 0.024 \\
Abnormal $(<32 \%)$ & $20.16 \pm 10.25$ & $22.09 \pm 8.89$ & $<0.001$ \\
Viability & & $30.20 \pm 5.09$ & a) \\
Normal $(\geq 58 \%)$ & $18.78 \pm 9.42$ & & $<0.001$ \\
Abnormal $(<58 \%)$ & $44.30 \pm 19.37$ & $22.57 \pm 9.32$ & 0.004 \\
Morphology & & $21.96 \pm 8.61$ & \\
Normal $(\geq 4 \%)$ & $19.03 \pm 9.88$ & & \\
Abnormal $(<4 \%)$ & $19.35 \pm 10.26$ & & \\
\hline
\end{tabular}

Values are presented as mean \pm standard deviation.

DFI, DNA fragmentation index.

a) A statistical analysis could not be done because there were too few samples with abnormal viability $(n=2)$.

As shown, the percentage of sperm showing progressive motility, viability, and normal morphology significantly decreased after thawing. In particular, progressive motility before freezing was generally two-fold greater than the progressive motility index after thawing $(32.78 \% \pm 12.38 \%$ and $16 \% \pm 9.08 \%$, respectively). Although the viability rate after cryopreservation was lower than that of fresh sperm, the mean of the post-thaw viability values was still over $50 \%$. SimilarIy, the normal morphology rate dropped from $3.87 \% \pm 2.01 \%$ to $2.55 \% \pm 1.45 \%$. In addition, the head defect rate and the midpiece or tail defect rate increased after conventional freezing. Of note, a statistically significant increase was observed in the post-thawing DFI (compared with the pre-freezing values).

\section{Effects of conventional freezing on DFI related to semen parameters}

The post-thawing DFl increased in all groups defined by abnormal or normal values of all the parameters. These differences were generally statistically significant (Table 5). A statistical analysis of the increased DFI in the group with abnormal viability group after freezing could not be done because there were too few samples with abnormal viability $(n=2)$.

\section{Discussion}

The DFI gives more information on men's reproductive potential than other parameters. Moreover, sperm with fragmented DNA have been found to be involved in failure of fertility and pregnancy loss [18]. As a result, interest has emerged in identifying causes of sperm DNA damage and establishing relationships between DNA fragmentation and conventional semen parameters. Many studies have reported correlations of DNA fragmentation with certain individuallevel characteristics and semen parameters. We found that the DFI did not significantly vary across groups with different characteristics.

Since conventional freezing is a technique that can preserve sperm for a long time, it can be an important component of the treatment of male infertility in ART. Because the processes of freezing and thawing are thought to damage both sperm structure and function, it is important to be aware of the effects cryopreservation may have on features of sperm that are important for egg fertilization, such as DNA content, acrosomal integrity, motility, and viability $[2,19]$. Cryopreservation has been shown to cause significant decreases in sperm parameters, including progressive motility, viability, and normal morphology [4]. Di Santo et al. [10] suggested that the damage caused 
by the freezing and thawing process may be related to methodological inadequacies, such as difficulties in controlling the freezing temperature drop curve. Others have proposed that these decreases are due to damage during the freezing and thawing processes from elements such as thermal shock, which causes formation of intracellular and extracellular ice crystals, cellular dehydration, and osmotic shock [20]. As a result of cryopreservation procedures, the susceptibility to lipid peroxidation (LPO) increases because more reactive oxygen species are produced. Therefore, sperm cells that are sensitive to LPO must tolerate stress at their membranes [21]. The formation of intracellular or extracellular ice crystals results from rapid cooling, which causes breaches in membranes and affects organelle function [10]. Moreover, the efflux of water from the internal to the external environment increases the concentration of solutes and osmotic pressure, with consequential toxicity damage, dehydration, and cell volume changes. This also occurs during the thawing process when ice melts or recrystallizes [22]. The greater the temperature increase, the greater the damage caused by recrystallization [23].

Our study had results similar to other reports, including the observation of a significant decrease in the progressive motility and survival rate after thawing $[2,10,24]$. However, the degree to which these parameters decrease has shown variation according to the use of various cryopreservation/thawing methods or CPAs. In many previous studies, motility after thawing decreased by $20 \%$ to $50 \%$ following different cryopreservation methods [25-27]. The decrease in sperm motility that we found may have been due to cryopreservation-induced damage to the mitochondrial membrane.

We also found that sperm viability was lower after thawing than before freezing. This effect may be associated with structural changes in membranes during the freezing and thawing processes. Ice crystals, which result in membrane leakage and impaired organelle function, causes a decrease in viability [10]. In the literature, plasma membrane destruction in the tail region has been suggested to be a probable reason for tail defects. Ozkavukcu et al. [19] showed that an increase in the frequency of coiled tails usually occurred after osmotic changes. The effects of low temperatures on membrane lipid structure and transmembrane water canal proteins have been studied. The addition or removal of CPA and changes occurring in water content during freezing may lead to tail coiling [19]. The formation of reactive oxygen species is one of the major deleterious effects of the procedure. In our study, native sperm were used, allowing any antioxidant features of seminal plasma to exert their protective effect [19].

In our study, glycerol was used as a cryoprotectant in the freezing protocol. Glycerol has a certain effect on motility, although freezing and thawing seem to cause more damage $[28,29]$. Because there is a strong correlation between the increase in immotile sperm and the decrease in viability after thawing, the decrease in motility may be due to the loss of viability [19]. Glycerol acts by decreasing the freezing point of a substance, reducing the concentration of salts and solutes in the sample, and decreasing ice formation in sperm. Furthermore, it supports the membrane structure, permeability, and stability of the lipid bilayer, the association of surface proteins, and cellular metabolism [10]. However, glycerol can cause poor outcomes in terms of membrane and acrosome structure. Furthermore, a variety of undesirable effects may result from using glycerol as a cryoprotectant, such as acrosomal internal membrane alteration, the presence of an undulating membrane, nucleus inhomogeneity, and disorganization in mitochondrial crests. Thus, during freezing, the cells may be partially damaged, bringing about a decrease in sperm parameters and harm to DNA integrity.

We found a significant difference in the DFI after conventional freezing. Both normal and abnormal groups defined in terms of progressive motility, viability, and morphology showed statistically significant increases in the DFI when comparing the observations made before freezing with those made after thawing. This result demonstrates that the conventional freezing procedure can damage DNA integrity. This hypothesis has been controversial for many years. Some authors have reported that DNA fragmentation after cryopreservation increased [27,30]. According to Spano et al. [31], sperm quality (including sperm DNA integrity assessed by SCSA) worsens after thawing. Similarly, some authors compared the DFI before freezing and after thawing by the TUNEL assay, and reported that cryopreservation negatively affected DNA integrity [32]. On the contrary, Lusignan et al. [8] reported that freezing and thawing did not damage sperm chromatin integrity. Isachenko et al. [33] also concluded that DNA fragmentation did not increase after thawing.

Some studies have reported that freezing and thawing produce DNA damage to different degrees in different subjects. Donnelly et al. [34] concluded that spermatozoa from fertile men seemed to be more resistant to damage than samples from infertile men. Others have shown no significant decrease in DNA integrity after freezing [35]. It is known that poor-quality sperm due to reduced protamination contains partially decondensed chromatin. Chromatin condensation may produce functional immaturity [34]. In contrast, some authors have concluded that sperm DNA fragmentation does not result from the freezing and thawing procedure. Isachenko et al. [33] compared the effects of slow freezing and vitrification on sperm DNA integrity in the absence of a CPA. They found that cryopreservation did not damage DNA integrity. Similar results were also reported by Duru et al. [36], who found that cryopreservation was associated with disruption of the mitochondrial membrane potential and activation of caspase 3, 8, and 9, but had no significant effect on DNA fragmentation. Caspase 3 is an important marker of the entry of a cell into the 
apoptotic pathway. Cryopreservation increases its activation, leading to DNA fragmentation [37]. Some studies have also revealed relationships between caspase activation, phosphatidylserine externalization, and DNA fragmentation [38].

Although the Halosperm test has recently become popular $[39,40]$ because it is simple, readily available, and a cost-effective choice for assessing DNA fragmentation [16], and it does not require expensive and complex instrumentation and can be performed using equipment available in andrology laboratories, there are still some limitations involving its techniques. The Halosperm test is an indirect technique measuring the amount of halos formed when nuclear proteins are removed, which is directly correlated to single-stranded DNA damage [41]. Furthermore, the sperm analyzed by the test cannot be used, especially for intracytoplasmic sperm injection. Additionally, it was reported that results from the SCD test in some circumstances depend on observers in a subjective fashion [42]. However, the use of standard categories for the halos may reduce interobserver variability. Another disadvantage is that the Halosperm test is not recommended for patients with severe oligozoospermia or cryptozoospermia. Furthermore, although there are several methods of performing DNA fragmentation assays, the Halosperm test was selected for this study because it is a validated, improved version of the SCD test. Chohan et al. [43] found that the TUNEL, SCD, acridine orange, and SCSA tests were significantly correlated, so that it is thought that sperm integrity tests generally correlate moderately with each other. In conclusion, the present study revealed significant effects of conventional freezing on sperm progressive motility, viability, morphology, and DNA fragmentation. The cryoinjury mechanism in the cryopreservation of human sperm requires further study.

\section{Conflict of interest}

No potential conflict of interest relevant to this article was reported.

\section{Acknowledgments}

We sincerely thank Mr. Elihu Welber of San Anselmo, CA, USA, from Health Volunteers Overseas (HVOUSA), for his excellent English editing.

\section{ORCID}

\section{Minh Tam Le}

Thai Thanh Thi Nguyen

Tung Thanh Nguyen

Trung Van Nguyen

Tam An Thi Nguyen
Quoc Huy Vu Nguyen

Thanh Ngoc Cao https://orcid.org/0000-0002-4744-7059

https://orcid.org/0000-0001-6362-0934

\section{Author contributions}

Conceptualization: MTL, TTTN, QHVN, TNC. Data curation: MTL, TTTN, TVN. Formal analysis: TTTN, TVN. Methodology: MTL, TTTN, TVN, TTN, TATN. Project administration: MTL. Visualization: TTTN, TTN, TVN. Writing - original draft: MTL, TTTN. Writing - review \& editing: MTL, TTTN, TTN,TVN, QHVN, TNC.

\section{References}

1. World Health Organization. Laboratory manual of the WHO for the examination of human semen and sperm-cervical mucus interaction. Ann Ist Super Sanita 2001;37:I-XII, 1-123.

2. Le MT, Nguyen TT, Nguyen TT, Nguyen VT, Nguyen TT, Nguyen $\mathrm{VQ}$, et al. Cryopreservation of human spermatozoa by vitrification versus conventional rapid freezing: effects on motility, viability, morphology and cellular defects. Eur J Obstet Gynecol Reprod Biol 2019;234:14-20

3. Colas C, Junquera C, Perez-Pe R, Cebrian-Perez JA, Muino-Blanco T. Ultrastructural study of the ability of seminal plasma proteins to protect ram spermatozoa against cold-shock. Microsc Res Tech 2009;72:566-72.

4. Hammadeh ME, Askari AS, Georg T, Rosenbaum P, Schmidt W. Effect of freeze-thawing procedure on chromatin stability, morphological alteration and membrane integrity of human spermatozoa in fertile and subfertile men. Int J Androl 1999;22:15562.

5. Isachenko V, Isachenko E, Katkov II, Montag M, Dessole S, Nawroth $\mathrm{F}$, et al. Cryoprotectant-free cryopreservation of human spermatozoa by vitrification and freezing in vapor: effect on motility, DNA integrity, and fertilization ability. Biol Reprod 2004;71:1167-73.

6. Rahiminia T, Hosseini A, Anvari M, Ghasemi-Esmailabad S, Talebi AR. Modern human sperm freezing: effect on DNA, chromatin and acrosome integrity. Taiwan J Obstet Gynecol 2017;56:472-6.

7. Schulte RT, OhI DA, Sigman M, Smith GD. Sperm DNA damage in male infertility: etiologies, assays, and outcomes. J Assist Reprod Genet 2010;27:3-12.

8. Lusignan MF, Li X, Herrero B, Delbes G, Chan PT. Effects of different cryopreservation methods on DNA integrity and sperm chromatin quality in men. Andrology 2018;6:829-35.

9. Muriel L, Garrido N, Fernandez JL, Remohi J, Pellicer A, de los Santos MJ, et al. Value of the sperm deoxyribonucleic acid fragmentation level, as measured by the sperm chromatin disper- 
sion test, in the outcome of in vitro fertilization and intracytoplasmic sperm injection. Fertil Steril 2006;85:371-83.

10. Di Santo M, Tarozzi N, Nadalini M, Borini A. Human sperm cryopreservation: update on techniques, effect on DNA integrity, and implications for ART. Adv Urol 2012;2012:854837.

11. Punyatanasakchai $P$, Sophonsritsuk $A$, Weerakiet $S$, Wansumrit $S$, Chompurat D. Comparison of cryopreserved human sperm in vapor and liquid phases of liquid nitrogen: effect on motility parameters, morphology, and sperm function. Fertil Steril 2008;90: 1978-82.

12. Agha-Rahimi A, Khalili MA, Nabi A, Ashourzadeh S. Vitrification is not superior to rapid freezing of normozoospermic spermatozoa: effects on sperm parameters, DNA fragmentation and hyaluronan binding. Reprod Biomed Online 2014;28:352-8.

13. Slabbert M, du Plessis SS, Huyser C. Large volume cryoprotectant-free vitrification: an alternative to conventional cryopreservation for human spermatozoa. Andrologia 2015;47:594-9.

14. Sanchez-Alvarez J, Cano-Corres R, Fuentes-Arderiu X. A complement for the WHO laboratory manual for the examination and processing of human semen (First Edition, 2010). EJIFCC 2012; 23:103-6.

15. Tandara M, Bajic A, Tandara L, Bilic-Zulle L, Sunj M, Kozina V, et al. Sperm DNA integrity testing: big halo is a good predictor of embryo quality and pregnancy after conventional IVF. Andrology 2014;2:678-86.

16. Fernandez JL, Muriel L, Goyanes V, Segrelles E, Gosalvez J, Enciso $M$, et al. Halosperm is an easy, available, and cost-effective alternative for determining sperm DNA fragmentation. Fertil Steril 2005;84:860.

17. Paoli D, Lombardo F, Lenzi A, Gandini L. Sperm cryopreservation: effects on chromatin structure. Adv Exp Med Biol 2014;791:13750.

18. Aitken RJ, De luliis GN, McLachlan RI. Biological and clinical significance of DNA damage in the male germ line. Int J Androl 2009;32:46-56.

19. Ozkavukcu S, Erdemli E, Isik A, Oztuna D, Karahuseyinoglu S. Effects of cryopreservation on sperm parameters and ultrastructural morphology of human spermatozoa. J Assist Reprod Genet 2008;25:403-11.

20. Stanic P, Tandara M, Sonicki Z, Simunic V, Radakovic B, Suchanek E. Comparison of protective media and freezing techniques for cryopreservation of human semen. Eur J Obstet Gynecol Reprod Biol 2000;91:65-70.

21. Amor H, Zeyad A, Alkhaled Y, Laqqan M, Saad A, Ben Ali H, et al. Relationship between nuclear DNA fragmentation, mitochondrial DNA damage and standard sperm parameters in spermatozoa of fertile and sub-fertile men before and after freeze- thawing procedure. Andrologia 2018;50:e12998.

22. Said TM, Gaglani A, Agarwal A. Implication of apoptosis in sperm cryoinjury. Reprod Biomed Online 2010;21:456-62.

23. Mazur P, Rall WF, Rigopoulos N. Relative contributions of the fraction of unfrozen water and of salt concentration to the survival of slowly frozen human erythrocytes. Biophys J 1981;36: 653-75.

24. Oberoi B, Kumar S, Talwar P. Study of human sperm motility post cryopreservation. Med J Armed Forces India 2014;70:349-53.

25. Isachenko E, Isachenko V, Weiss JM, Kreienberg R, Katkov II, Schulz $\mathrm{M}$, et al. Acrosomal status and mitochondrial activity of human spermatozoa vitrified with sucrose. Reproduction 2008; 136:167-73.

26. Satirapod C, Treetampinich C, Weerakiet S, Wongkularb A, Rattanasiri S, Choktanasiri W. Comparison of cryopreserved human sperm from solid surface vitrification and standard vapor freezing method: on motility, morphology, vitality and DNA integrity. Andrologia 2012;44 Suppl 1:786-90.

27. Hosseini A, Khalili MA, Talebi AR, Agha-Rahimi A, Ghasemi-Esmailabad S, Woodward B, et al. Cryopreservation of low number of human spermatozoa; which is better: vapor phase or direct submerging in liquid nitrogen? Hum Fertil (Camb) 2019;22:12632.

28. Rofeim O, Gilbert BR. Long-term effects of cryopreservation on human spermatozoa. Fertil Steril 2005;84:536-7.

29. Desrosiers $P$, Legare $C$, Leclerc $P$, Sullivan R. Membranous and structural damage that occur during cryopreservation of human sperm may be time-related events. Fertil Steril 2006;85:1744-52.

30. Chohan KR, Griffin JT, Carrell DT. Evaluation of chromatin integrity in human sperm using acridine orange staining with different fixatives and after cryopreservation. Andrologia 2004;36:321-6.

31. Spano M, Cordelli E, Leter G, Lombardo F, Lenzi A, Gandini L. Nuclear chromatin variations in human spermatozoa undergoing swim-up and cryopreservation evaluated by the flow cytometric sperm chromatin structure assay. Mol Hum Reprod 1999;5:2937.

32. de Paula TS, Bertolla RP, Spaine DM, Cunha MA, Schor N, Cedenho AP. Effect of cryopreservation on sperm apoptotic deoxyribonucleic acid fragmentation in patients with oligozoospermia. Fertil Steril 2006;86:597-600.

33. Isachenko E, Isachenko V, Katkov II, Rahimi G, Schondorf T, Mallmann $P$, et al. DNA integrity and motility of human spermatozoa after standard slow freezing versus cryoprotectant-free vitrification. Hum Reprod 2004;19:932-9.

34. Donnelly ET, McClure N, Lewis SE. Cryopreservation of human semen and prepared sperm: effects on motility parameters and DNA integrity. Fertil Steril 2001;76:892-900. 
35. Evenson DP, Melamed MR. Rapid analysis of normal and abnormal cell types in human semen and testis biopsies by flow cytometry. J Histochem Cytochem 1983;31(1A Suppl):248-53.

36. Duru NK, Morshedi M, Schuffner A, Oehninger S. Cryopreservation-thawing of fractionated human spermatozoa and plasma membrane translocation of phosphatidylserine. Fertil Steril 2001;75:263-8.

37. Grunewald S, Sharma R, Paasch U, Glander HJ, Agarwal A. Impact of caspase activation in human spermatozoa. Microsc Res Tech 2009;72:878-88.

38. Karabulut S, Demiroglu-Zergeroglu A, Yilmaz E, Kutlu P, Keskin I. Effects of human sperm cryopreservation on apoptotic markers in normozoospermic and non-normozoospermic patients. Zygote 2018;26:308-13.
39. Henkel R. Clinical utility of sperm DNA fragmentation testing: a commentary. Transl Androl Urol 2017;6(Suppl 4):S632-5.

40. Vandekerckhove F. Guidelines on sperm DNA fragmentation testing. Transl Androl Urol 2017;6(Suppl 4):S586-7.

41. Panner Selvam MK, Agarwal A. A systematic review on sperm DNA fragmentation in male factor infertility: laboratory assessment. Arab J Urol 2018;16:65-76.

42. Agarwal A, Majzoub A, Esteves SC, Ko E, Ramasamy R, Zini A. Clinical utility of sperm DNA fragmentation testing: practice recommendations based on clinical scenarios. Transl Androl Urol 2016;5:935-50.

43. Chohan KR, Griffin JT, Lafromboise M, De Jonge CJ, Carrell DT. Comparison of chromatin assays for DNA fragmentation evaluation in human sperm. J Androl 2006;27:53-9. 